in no way alter the apsidal progress determined by Einstein from (I). The "measuring rod," however, does not now alter in length for different orientations, which is a somewhat comforting result.

The gravitational potentials in (2) are not additive, and Prof. Eddington ("Report on Relativity," p. 59) proposes to get over the difficulty by neglecting squares of $m / r_{1}$ in (2), which would then give

$$
\begin{aligned}
d s^{2}=-\left(\mathrm{I}+\frac{2 m}{r_{1}}\right)\left\{\delta r_{1}^{2}+r_{1}^{2} \delta \theta^{2}+r_{1}^{2} \sin ^{2} \theta \delta \phi^{2}\right\} \\
+\left(\mathrm{I}-\frac{2 m}{r_{1}}\right) \delta t^{2} .
\end{aligned}
$$

so that the contributions of potential would be additive.

Unfortunately, neglecting squares of $m$ leads to a change in the apsidal progress, and it appears that treating (3) as exact gives $4 / 3$ times the apsidal progress calculated from (I). We cannot, therefore, neglect squares of $m$ at an early stage without violating the observations which (I) or (2) was called in to explain. The adjustment is, in fact, so delicate in (I) that we may not approximate at all until the end of the calculation.

What, then, are the exact equations for two finite bodies $m_{1}$ and $m_{2}$, both mobile? Here we are not permitted to superpose any velocity which would reduce one of the bodies to rest.

Portsmouth, March 30.

I AM indebted to the Editor's courtesy for the opportunity to comment on the foregoing letter. In deciding whether an approximation is allowable, regard must be had to the problem to which it is to be applied. It is true that equation (3) neglects a term of importance in the motion of the apse, and is therefore not valid for the problem of the perihelion of Mercury; but there may be other problems for which the approximation can be justified. One of these is the calculation of the $\mathrm{G}_{\mu \nu}$ for continuous matter on p. 59 of my "Report." My proof starts with the approximate calculation of the line-element in a sphere which is ultimately made infinitely small; I think that the justification of the neglect of $\mathrm{m}^{2}$ iven in $\$ 36$ is correct, though the argument is intricate, and I would welcome detailed criticism. But, for example, my formulæ are not sufficiently accurate to give the rotation of the apse-line of a particle moving freely through a diffuse spherical nebula.

Dr. Walker goes on to ask: What are the exact equations for two particles moving freely? He who can supply the answer will have solved one of the biggest mathematical problems of the theory. The problem of two bodies in Einstein's theory is an outstanding challenge, like the problem of three bodies in Newton's theorv. The solution will give $d s^{2}$ throughout all time, and therefore incidentally the tracks of the particles which are the singularities of the solution. I am not satisfied that it has yet been proved that the tracks are periodic-that there is no dissipation of energy by the gravitational waves set up.

Observatory, Cambridge, April 2.

The success of Prof. Bohr's theory, and of Sommerfeld's developments of it, is' sufficient evidence of the truth of its general assumptions, and therefore of the reality of the ideas on which it is based. "Fixed electron" theories have nothing to set against (e.g.) the weighing of the helium atom by means of its spectrum or the detailed prediction of the structure of the L-rays; moreover, those theories, as Prof. Bohr points out, are empirical and based on no general principle. But the superiority of the "orbital electrons" theory does not alter the fact that there are things which it is very difficult to reconcile with the view that the stationary states of an atom consist of electronic orbits of which the dimensions are comparable with I $\AA$., and of which the periods are comparable with Io $^{-16} \mathrm{sec}$.

The suggestion that I made is that, by means of a generalised principle of correspondence, the distinction between moving and fixed electrons might be abolished and the conceptions that have proved so fruitful in explaining spectra made available immediately for explaining also such things (if there are such things) as are only explicable by fixed electrons. Thus the distinction would be abolished if "time" had no meaning inside the atom. For the difference between electrons following an orbit and electrons fixed at points on that orbit can only be expressed in terms of temporal conceptions; if all such conceptions are tetally invalid in dealing with problems of atomic st ucture the distinction vanishes.

Expressed in the very crude form demanded by brevity, such a suggestion will doubtless be deemed unacceptable, or even unintelligible. Here I would only mention two considerations, one special and the other general, that have led to it. First, very difficult questions can be asked (and have been asked by Stark) concerning what happens in the interval during which an atom passes from one stationary state to another, and during which it emits or absorbs homogeneous radiation. We might deny that such questions have any meaning, because there is no such thing as an interval during which the transition takes place. It is not merely that the interval is infinitesimally small or zero; it is that the conception of a time interval is not permissible when we are considering the process which we observe as change of radiant energy and explain as change of atomic structure. Secondly, the conception of continuity is very closelv associated with that of time. The assumption of the older physics, that all fundamental theories (usually mistermed "laws") were to be expressed by means of differential equations, involved in all but a few instances (which can be explained awav) that the variable with respect to which the integration of the equations was to be made, in order to compare the theory with experiment, was the "time." Now it is the characteristic and essential feature of Prof. Bohr's theory that the emission and absorption of homogeneous radiation, which is the outward expression of change of atomic state, is not to be described by a differential equation. Consistency seems to compel us to conclude that it is also not to be described ultimately in terms of con-

\title{
Atomic Structure.
}

My letter in NATURE of November 25 last has served a useful purpose by evoking the very interesting account of his new line of work which Prof. Bohr has given in the issue of March 24. But since he did not deal, and scarcelv professed to deal, with my suggestion, perhaps I may try to make clearer what that suggestion is.

NO. 2684 , VOL. IO7] ceptions in which "time" plays any part.

Norman R. CAMpbel.L.

\section{S. EdDington.}

\section{British Plants Available as a Source of Industrial Alcohol.}

THE production of cheap alcohol for industrial purposes is a subject much under discussion at the present time, and in considering the question of available materials from which it could be obtained the following notes may be of interest.

Apart from the mangel and sugar-beet, it is im- 